\title{
4 Aristoteles oder warum Rhetorik nützlich ist
}

\section{0 „Der Mensch ist ein Wesen, das spricht“}

Warum die Mönche schweigen, haben wir in Kapitel $3 \mathrm{zu}$ beantworten versucht: aus den gleichen Gründen, aus denen auch Philosophen das Schweigen favorisieren; beide möchten der Quelle unverstellter Wahrheit näherkommen, und das erreicht man im traditionellen Verständnis durch kontemplatives Schweigen, insofern sich dadurch Menschen am ehesten, sei es schauend oder hörend, für die Erfahrung von Transzendenz zu öffnen vermögen. Entsprechend gilt (bzw. galt) die kontemplative Lebensweise als die höchste und beglückendste Form menschlicher Existenz (s. o. Kap. 3.1; Pieper 2012 und Wetz 1994, 102 ff.). Welche Folgen diese Hochschätzung der „,vita contemplativa“ hatte, ist mit wenigen markanten Strichen präzis von Sloterdijk unter dem Stichwort „Weltverleumdung“ skizziert worden, der in der Tradition des besonders von Nietzsche nobilitierten Verdachts gegenüber der „alteuropäischen Theoriekultur“ der Herkunft des „homo theoretikus“ nachgegangen ist mit der Frage, ob dieser „homo wirklich aus so gutem Hause ist, wie er selbst von seinen ersten Tagen an versichert“ (2010, 63). Das Ergebnis dieses Frageinteresses ist erwartungsgemäß mehr als ernüchternd, insofern es einen „fatalen“ Reduktionismus und eine „pathetische Verarmung aller Weltbezüge“ freilegt, die Sloterdijk in Analogie zu „iatrogenen“ von „philosophogenen Krankheiten [...] der abendländischen Rationalitätskultur“ reden lässt, deren Spuren bis in die Gegenwart reichen (2010, $102 \mathrm{ff}$.).

Natürlich trifft diese Einschätzung der „fatalen“ Folgen der „alteuropäische Theoriekultur“ auch für die Rhetorik in vollem Umfang zu, weil „Weltverleumdung“ und „Rhetorikverleumdung“ (Toulmin 1986) immer recht nah beieinander gewohnt haben. Dennoch! Es gibt bzw. es gab einen antiken Philosophen der Meisterklasse, der trotz seiner Zugehörigkeit zu dieser „alteuropäischen Theoriekultur" für die Rhetorik ein Glücksfall war, wie ich zeigen zu können hoffe, ich meine natürlich Aristoteles. Zwar zählt auch für ihn Kontemplation zur wertvollsten Tätigkeit des Menschen und entsprechend hatte sie als „bios theoretikos“ den Primat innerhalb der Hierarchie möglicher Lebensformen („bioi“) inne (vgl. König 1998; Ritter 1977, 9 ff.; Arendt 1960, 12 ff., 282 ff.), doch als dauernde Existenzweise behielt sie Aristoteles den Göttern vor, während er die Menschen nur selten und nur kurzfristig an ihr teilhaben ließ. Darum wählte Aristoteles auch nicht das kontemplative Schweigen zur spezifizierenden Kennzeichnung und gleichzeitigen Auszeichnung des Menschen, sondern eine Kompetenz, die Schweigen überhaupt erst möglich macht und die dort und nur dort gebraucht wird, wohin Platon im oben erläuterten Höhlengleichnis (Kap. 3.2) die widerwilligen Philosophen zurückschickt, nämlich dorthin, wo „menschliche Dinge 
betrieben [werden]“ und d.h.: wo, um sie betreiben $\mathrm{zu}$ können oder gar gut betreiben $\mathrm{zu}$ können, unbedingt geredet werden muss. Entsprechend lautet eine der berühmtesten und für die europäische Denktradition folgenreichsten einschlägigen Aussagen über den Menschen bei Aristoteles auch: „Der Mensch ist ein Wesen, das logos hat“ (zoon logon echon, Polit. 1253; Schütrumpf 1991/1, 212 ff.; Krautter 2009; Taylor 2017).

\subsection{Der evolutionäre Vorteil der Sprache}

Bei der Übersetzung des griechischen Kernwortes „logos“ tat sich schon die lateinische Sprache schwer, die mit der gängigen Formel vom „animal rationale“ (Vernunftwesen) (Schmidinger und Sedmak 2004; Schnädelbach 1984; 1992) erkennbar nur einen Teilaspekt des „logos“-Begriffs erfasste. Erst recht blieb nicht nur für Goethes „Faust“ im Studierzimmer die Frage unentscheidbar, wie man im Deutschen den Begriff „logos“ (in diesem Fall aus dem biblischen Johannes-Prolog: „en arche en ho logos“) angemessen übersetzen könne, ohne in interpretierende Hilfsübersetzungen ausweichen $\mathrm{zu}$ müssen wie „sprachfähige Vernunft“ bzw. „vernunftfähige Rede“. Unbeeindruckt von dieser aporetischen Tradition wartet dagegen Heidegger in seiner (unten in Kap. 7 noch genauer zu erläuternden) Aristoteles-Vorlesung von 1924 mit einem forschen Übersetzungsvorschlag für das eben erwähnte Aristoteles-Zitat auf: „Der Mensch ist ein Wesen, das spricht“ (2002, 107 und öfter). Unbekümmertheit ist Heidegger im Umgang mit griechischen Texten bekanntlich nie ganz fremd, doch in diesem Fall bringt sie einen Teilaspekt des „logos“-Begriffs ins Spiel, der an der betreffenden Bezugsstelle der Aristotelischen Politik fraglos am ehesten deren Diskussionsfokus trifft. Außerdem ist dieser Teilaspekt für unser Frageinteresse in diesem Kapitel besonders geeignet, um nach dem Aufenthalt in den Höhen anachoretischer Kontemplation die Rückkehr in die VodafoneWelt zu versuchen, deren Bewohner - so der Ertrag aus Kap. 3 - aufgrund erodierter Traditionsprämissen nicht das Reden, sondern eher das Schweigen für erklärungs- und legitimationsbedürftig halten dürften. Die Heidegger'sche Übersetzung von „zoon logon echon“ ist für dieses Frageinteresse besonders geeignet, weil sie sich als Abstützung geradezu anbietet für eine kühne These, die oben (Kap. 2) bereits erwähnt wurde und jetzt noch genauer zu begründen bleibt, nämlich: dass Aristoteles der Vodafone-Behauptung, ,wir [seien] nicht auf der Welt, um zu schweigen“, sicher gern zugestimmt hätte. Er hätte diesem Satz sogar zustimmen müssen, weil man einem Wesen, dessen auszeichnendes Merkmal das Sprechen-Können sein soll, ja schwerlich auf Schweigen als Sinn seiner Existenz verpflichten kann. 
Freilich ist das auch fast alles, was sich für unser Frageinteresse aus Heideggers Aristoteles-Vorlesung gewinnen lässt; denn die zieht zwar die Aristotelische Rhetorik in singulärer Weise als Materialquelle heran, doch das tut sie nicht, um das Aristotelische Verständnis von Rhetorik zu klären (obwohl sich attraktive Einlassungen durchaus finden lassen), sondern um die Grundbegriffe der aristotelischen Philosophie (so der Titel von Heideggers Vorlesung) in gezielter Abgrenzung gegen die Rhetorik und deren notorische Verfallstendenz (,Verfallensgeneigtheit") $\mathrm{zu}$ rekonstruieren (2002, 108 und öfter). Insofern geht es im Folgenden auch darum, die Heidegger'sche Übersetzung von „zoon logon echon“ mit „der Mensch ist ein Wesen, das spricht“ vor Heideggers eigener Interpretation zu schützen, um den authentischen Gehalt dieser Aussage für eine philosophische Rehabilitation der Rhetorik zu retten, die bei Aristoteles fraglos beginnt - mag diese Einschätzung auch erst retrospektiv aus einer seit Nietzsche versuchten philosophischen Rhetorik-Rehabilitation möglich sein (s. Kap. 8).

Der Schutz der Heidegger'schen Übersetzung vor Heideggers eigener Interpretation des übersetzen Aristotelischen Satzes muss bereits bei dessen Verständnis als „Definition“ beginnen. Natürlich erfüllt der Aristotelische Bezugstext wesentliche Merkmale einer Definition; doch die werden erst dann dominant, wenn der entsprechende Bezugstext vorgängig in einem Maße entkontextualisiert wird, dass dessen argumentative Funktion fast unkenntlich wird. Zwar verschweigt Heidegger nicht ganz, dass sich in dem von ihm „Definition“ genannten Satz über den Menschen bei Aristoteles „ein Grundcharakter des Daseins als zoon politikon offenbart“, insofern „der Mensch in der Weise des Miteinanderseins ist“, das „seine Grundmöglichkeit im [...] Miteinandersprechen hat“ (2002, 104); doch nicht für diese „politische“ Funktionalisierung des Sprechens als Medium praktischer Verständigungsarbeit interessiert sich Heidegger, sondern für „Sprechen“ als „Basis [...] der Begriffsbildung im Dasein“, d.h. für den nach Heidegger an Aristoteles beispielhaft ablesbaren Prozess der Befreiung philosophischer Begriffsbildung aus der meinungsverhafteten Alltagsgerede, in der seit Platon die Rhetorik pejorisierend verortet wird (2002, 108 und öfter; vgl. Kopperschmidt 2009, 318 ff. und unten Kap. 7). Durch dieses Frageinteresse wird aber die eigentliche Aristotelische Frageintention bis zur Unkenntlichkeit verstellt, die ja das Reden in Theorie und Praxis gerade an eine spezifische Lebensform (den bios politikos) systematisch rückbinden will, um es so mit ihr zusammen in einer Weise zu nobilitieren, die der Heidegger'schen Sichtweise der Rhetorik radikal entgegensteht. Dieses interpretative Kunststück gelingt Heidegger auch nur dadurch, dass er in einer abenteuerlichen Kompilationsstrategie das Aristotelische Rhetorikverständnis mit dem Platonischen in einer Weise vermittelt, die Aristoteles zum authentischen Exegeten der Platonischen Idee einer „positiven Rhetorik“ macht, die aber, wenn unsere einschlägige Interpretation (in Kap. 7) plausibel ist, nur eine 
sozialtechnologische Restringierung der Rhetorik betreibt. Statt diesen verqueren Konstrukten hier schon weiter nachzugehen zu wollen, sei kurz der authentische Aristotelische Reflexionskontext skizziert, um die wirkliche Funktionsbeziehung zu klären, die zwischen den beiden Kernaussagen der Politik besteht, nämlich dass der Mensch sowohl „ein sprechendes Wesen“ wie ein „politisches Wesen [meine Hervorhebung, J. K]“ sei (Polit. 1352/53). ${ }^{39}$ Dieses „sowohl als auch“ ist bei Aristoteles funktional genauerhin in der Weise bestimmt, dass der Mensch deshalb ein „sprechendes Wesen“ sein müsse, damit er ein „politisches Wesen“ sein könne; denn dazu habe ihm als einzigem Lebewesen die „Natur, die nichts umsonst tut“ (!), die Sprache gegeben, dass er in einer „polis“-Gemeinschaft zu leben vermöchte. Deshalb kann Schweigen keine politische Tugend sein und jeder, der „a-polis“ lebt, der also nicht in eine solche soziopolitischen Gemeinschaft eingebunden ist, muss „entweder mehr oder weniger als ein Mensch sein“, mithin „entweder Gott oder Tier“.

Wem bei der Lektüre dieser großartigen Textpassage (nicht zu Unrecht) darwinistisch oder sonst wie motivierte Bedenken gegen die Unterstellung eines teleologischen (bzw. finalistischen) Naturbegriffs kommen, ${ }^{40}$ den mag die Versicherung beruhigen, dass auch eine evolutionstheoretische Übersetzung des eben genannten Aristotelischen Teleologie-Theorems („Die Natur tut nichts umsonst“, vgl. dazu Schütrumpf 1991/1, 206 ff.; Wetz 1994, 164) dessen substanziellen Gehalt nicht eo ipso dementieren muss; denn dass die Sprachfähigkeit des Menschen ein ihn auszeichnendes Alleinstellungsmerkmal ist, erlaubt nicht nur, ihn in der sprachhumanistischen Tradition Herders zu einem „Geschöpf der Sprache“ zu machen $(1965,80)$, sondern ebenso, diese Sprachkompetenz als einen eminent wichtigen, wenn nicht sogar als den wichtigsten evolutionären Selektionsvorteil für die Hominisation des Menschen zu bestimmen. Um für diese heute kaum noch bestreitbare evolutionstheoretische Denklogik das Aristotelische Theorem retten zu können, muss eigentlich nur (mag dieses „nur“ auch geistesgeschichtlich viel wiegen) die teleologische Funktionsbeziehung zwischen Sprache (Logizität) und soziopolitischer Lebensform (Polizität) kausal reformuliert bzw. transformiert

39 Vgl. auch Aristoteles NE 1269b, außerdem Höffe, Otfried. Der Mensch. Ein politisches Tier? Stuttgart: Reclam, 1992; Schmidinger und Sedmak 2005; Zinsmaier 2009. Kritisch zu solch substanziellen Aussagen Arendt 1993, 11 und Meyer 1994, 197 ff.

40 Die einschlägige Diskussion ist neu entbrannt unter dem Titel „intelligent design“, womit eine dem Kreationismus verwandte Argumentation gemeint ist, die bestreitet, dass sich die komplexen Bedingungen möglichen Lebens durch Mutations- und Selektionsprozessen hinreichend erklären ließe. Vgl. u. a. Hemminger, Hansjörg. Und Gott schuf Darwins Welt. Gießen: Brunnen, 2009, 140 ff. Dagegen bes. Dawkins 1996; s. auch FN 14! Zur Funktion der verwandten These, dass „die Natur nichts überflüssig tut“, bei Kant s. u. Kap. 5.4. 
werden: Der Mensch ist primär deshalb Mensch geworden, weil er ein sprachfähiges Wesen ist. ${ }^{41}$

Dass dieser evolutionären Vorteil sich exemplarisch an der Komplexitätssteigerung sprachlich vermittelter Kooperativität ablesen lässt, bestreitet auch Tomasello mit seinen preisgekrönten und viel gerühmten (vgl. Habermas' Preisrede auf Tomasello 2013, 166 ff.) Arbeiten zur Evolutionsanthropologie bzw. -biologie der Kooperation nicht (2009, 339 ff.), wenn er auch - anders als Aristoteles - sich dadurch nicht genötigt glaubt, die Sprache zur Bedingung möglicher Kooperation machen zu müssen und in einem teleologischen Anthropozentrismus zu verorten; denn nicht erst Sprache benennt für Tomasello das zentrale Distinktiv zwischen Mensch und nichtmenschlichen Primaten, sondern etwas, was ihm bei Experimenten an Kleinkindern aufgefallen ist und was er als ontogenetischen Schlüssel zur Phylogenese des Menschen zu nutzen versucht (2009; 2010; Siefer 2010; Sennett 2012): Was Kinder nämlich bereits im Alter von 12-14 Monaten von allen nichtmenschlichen Primaten prinzipiell, nicht bloß graduell unterscheidet und insofern ein menschliches Alleinstellungsmerkmal begründet, ist ihre bereits vorsprachlich (!) (wenn natürlich auch erst rudimentär) ausgebildete singuläre Fähigkeit zu „[kollektiv] geteilter [und nicht nur subjektiver] Intentionalität [meine Hervorhebung, J. K.]“ bzw. „Wir-Intentionalität“ (Tomasello 2009, 17 ff., 83 ff., 341 ff.). Damit sind kognitive, referenzielle, soziale, motivationale, normative usw. Gemeinsamkeiten gemeint, die kooperierende Subjekte miteinander teilen und die sie so zu einem „Wir“ werden lassen, was für mögliche Handlungskoordinationen natürlich einen enormen „Anpassungsvorteil“ impliziert. Selbst wenn - wie gesagt - Sprache nach Tomasello nicht die Quelle spezifisch menschlicher Kooperationskompetenz ist, sondern deren Folge (und darum auch bei Zeigegesten gegenüber Kleinkindern bereits vorsprachlich an deren Blickrichtung nachgewiesen werden kann (2009, 22-23, $347 \mathrm{ff}$.; 2010, 64-65)), bedeutet die sprachlich vermittelte Kooperation dennoch nicht nur eine quantitative Steigerung möglicher Kooperationschancen, sondern eine qualitative Veränderung ihres Komplexitätsgrades (2009, 19 ff., 341 ff.); denn mit Sprache werden natürlich die für Zeigegesten verbindlichen engen Raum- und Zeitbedingungen exponentiell entgrenzbar und die „geteilte Intentionalität“ auf „Gegenstände“ lenkbar, die nur als Vorstellungen präsentierbar, reflektierbar und deliberativ diskutierbar sind. Wenn bei Tomasello auch weder Aristoteles noch gar Rhetorik vorkommen, das oben zitierte Aristotelische Grundtheorem der

41 Vgl. Arendt 1993, 10: „Menschen sind nur darum zur Politik begabte Wesen, weil sie mit Sprache begabte Wesen sind“. Noch radikalere Argumentation bei Isokrates und Quintilian: Ohne Sprache bzw. ohne deren persuasiven Gebrauch wären politische Gemeinschaftsformen gar nicht zustande gekommen, s. Jackob 2005, 200; Andersen 2001, 274 ff. 
engen Beziehung zwischen Logizität und Polizität wäre mit seinen evolutionsbiologischen Kategorien ebenso leicht reformulierbar wie die Nützlichkeit einer Rhetorik plausibilisierbar zu machen wäre, die sich als Methodisierung einer sprachlich vermittelten und darum vernunftgestützten Handlungskoordination versteht; und Rhetorik kann das, weil sie die dafür nötige Fundamentalbedingung einer „geteilten Intentionalität“ als zustimmungsbezogene Verständigungsarbeit im Interesse besserer Kooperationschancen operativ elaboriert.

\subsection{Die vierfache Nützlichkeit der Rhetorik}

Mit „zustimmungsbezogener Verständigungsarbeit“ wird ein Rhetorikbegriff bestimmbar, der sich nicht nur aus der Aristotelischen Politik und Rhetorik rekonstruieren, sondern sich auch gegen das Konzept einer bloßen Konzessionsrhetorik abgrenzen lässt als dem vorrangigen Typ einer sozialtechnologischen Überredungsrhetorik, ob sie nun auf der Kanzel (Augustin) oder in der Höhle gebraucht wird (Platon), ob sie sich nun der Befreiung aus einem sündhaft verstrickten oder aus einem meinungshaft verdunkelten Leben andient. Bei solcher Abgrenzung der Aristotelischen Rhetorik darf freilich nicht unterschlagen werden, was oben bereits gesagt wurde, dass nämlich diese sprachlich mediatisierte politische Existenzweise gleichwohl nur die zweitbeste Lebensform in deren triadischer Hierarchisierung darstellt (vgl. König 1998, 1071 ff.). Man sieht es bei genauerem Hinblicken auch den Aristotelischen Aussagen über Rhetorik noch an, dass sie sich von den traditionellen Fußangeln der Platonischen Rhetorikverdächtigung noch nicht ganz befreit haben. Diese Fußangeln sollen deshalb im folgenden Versuch einer systematisch interessierten Rekonstruktion des Aristotelischen Rhetorikbegriffs auch nicht ausgeklammert werden (s. Abschnitte a und b), wenn der Fokus dieses Rekonstruktionsversuches auch mehr auf die innovativen Aspekte dieses Rhetorikbegriffs zielt (s. die Abschnitte c und d), die ihn überhaupt erst in die Vorgeschichte der modernen philosophischen Rhetorikrehabilitation einzureihen erlauben.

Unter den traditionellen Versatzstücken der Aristotelischen Verständnisses von Rhetorik gehört fraglos deren defizit- bzw. mängeltheoretische Begründung zu den vertrautesten: Sie ist unter dem Term Konzessionsrhetorik oben (Kap. 3.3/3.4) bereits zur Sprache gekommen, um die Platonische und Augustinische Legitimation von Rhetorik als gleichsam kompensatorische Reaktion auf die intellektuellen Defizite, Insuffizienzen bzw. Mängel zu bestimmen, die es den meisten Menschen verwehren, ohne zusätzliche persuasive Wirkungsstrategien Wahrheitsevidenzen eine Überzeugungskraft abgewinnen zu können. Entsprechend bestimmt auch Aristoteles Rhetorik als „nützlich“, weil sie 
a) mithilft, „die Vielen“ bzw. „die Menge“ wenigstens „zu überreden (peithein), notfalls auch mit emotionaler Einwirkung (pathos) und/oder sprachlich einnehmender Gestaltung (lexis), was, wären wir alle vernunftbestimmte Wesen, völlig ,überflüssig“ wäre (Rhet. 1404a). Aber die Menschen sind nun einmal nicht alle total vernunftbestimmt oder - so Quintilian gleichsinnig (IO II 17, 28) - philosophisch ambitioniert, weshalb sie sich nicht überzeugen lassen, „selbst wenn wir das sicherste Wissen hätten“ (Rhet. 1355a, 1357a, 1404a; vgl. Rapp 2002/2, 814-815). Die grammatische Form dieses Zitats erinnert zumindest an eine Option, die es eigentlich gar nicht mehr geben kann, weil es Rhetorik nach Aristoteles nur mit solchen Sachverhalten zu tun hat, die genau das definitiv ausschließen, was hier wenigstens aus argumentationsstrategischen Gründen denkbar bleibt, nämlich der Besitz von „sicherstem Wissen“. Der ist aber deshalb definitiv auszuschließen, weil wir über Sachverhalte, „die auch anders sein können [meine Hervorhebung, J. K.]“ - so Aristoteles’ umständlich ontologische Formel für den Kontingenzcharakter der „Praxis“ - prinzipiell kein sicheres, geschweige denn „sicherstes Wissen“ haben können (Rhet.1357a), wie es vergleichsweise im Bereich von „Theorie“ (im o.g. Sinne) möglich ist, die darum aber auch nichts bewegen bzw. verändern, sondern nur kontemplativ genießen kann (vgl. Riedel 1972, 87; Ritter 1977, 9 ff.). Doch genau wegen dieser Unmöglichkeit praktischer Gewissheitschancen bzw. wegen der Theorieunfähigkeit von Praxis ist ja überhaupt nur Rhetorik nach Aristoteles nötig, die entsprechend auch darum nützlich genannt werden darf, weil sie

b) eine Methode anbietet, das fehlende Wissen im Bereich der Praxis „deliberativ“ (bouleusis) bzw. diskursiv zu kompensieren. Erkennbar geht es jetzt nicht mehr um Rhetorik als bloßer Konzession an die Schwäche „der Vielen“, sondern um deren Kompensation, wobei die hier gemeinten Mängel aber - anders als die spätere Begriffskonvention will (s.u. Kap. 5) - nicht anthropologischer, sondern ontologischer Natur sind, weshalb Mainberger auch lieber von „Kontingenzbewältigung“ spricht (1987/1, 153 ff., 257 ff.). Rhetorik ist, weil sie es mit ontologisch defizitären Sachverhalten zu tun hat, deshalb selbst eine vergleichsweise defizitäre Angelegenheit, gleichsam ein „Verlegenheit[sprodukt]“ (Blumenberg 1981, 108), dem jede philosophische Dignität abgeht, zumindest solange ontologische Rangstufen als selbstevident unterstellbar sind. ${ }^{42}$ Diese Rangindikation

42 Erhellend ist in diesem Zusammenhang, dass Aristoteles - durchaus konsequent - die Poesie für höherrangiger und philosophischer hält als die Historiographie, weil nur sie Handlungen erfinden kann, die „vollkommen“ sind, insofern sie „allgemein“ geltender „Notwendigkeit und Angemessenheit“ gehorchen, statt sich wie die Historiographie mit dem „Besonderen“ geschichtlicher Fakten zu beschäftigen und sie nachzuzeichnen, Poetik, c.9. 
widerspricht aber nicht notwendig einer pragmatischen Wertschätzung der Rhetorik als „nützlich“, sondern sie erklärt nur deren historisch dauerhaft prekären Status. Den kann Rhetorik nämlich auch durch noch so strenge empirische Beobachtungsarbeit bzgl. rhetorischer Wirkungsbedingungen nie wettmachen, weil ihr Handicap kein Exaktheitsdefizit meint, sondern ein in ihrem Gegenstand selbst fundiertes Wertdefizit, das sich auch genau bestimmen lässt: Rhetorik hat es - anders als Kontemplation - mit Sachverhalten $\mathrm{zu}$ tun, die nicht streng wissenschaftlich zugänglich sind, weil sie nicht ewig, nicht unvergänglich, nicht unveränderbar und deshalb auch nicht vollkommen sein können; ${ }^{43}$ denn Vollkommenheit schließt Veränderbarkeit logisch aus, insofern jede Veränderung von Vollkommenheit immer nur deren Minderung meinen kann. Rhetorik ist daher, was ihren originären Gegenstand angeht, eigentlich nur negatorisch bestimmbar, wie es Aristoteles an der eben zitierten Stelle ja auch exakt tut: Rhetorik beschäftigt sich mit Sachverhalten, die auch „anders sein können“, also veränderbar (bzw. kontingent) sind, weshalb wir „für sie keine strenge Wissenschaft besitzen“ und entsprechend über sie auch „,kein gesichertes Wissen [meine Hervorhebung, J. K.]“ haben können (Rhet. 1357a).

Die eben aufgelisteten Negate negieren beispielhaft Eigenschaften, die die Rangindikation eines Sachverhaltes traditionell ausmachten und so auch den Wert einer Wissenschaft traditionell bestimmten. Selbst wenn das heute wissenschaftsimmanent weithin irrelevant sein dürfte und auch Philosophie (von den Wissenschaften ganz zu schweigen) sich nicht mehr über „die Suche nach dem Unwandelbaren“ (Dewey 1998, 30 ff.) definiert - es ist ja noch nicht ganz so lange her, dass diese Suche und mit ihr die traditionelle Suche nach Gewissheit (so der einschlägige Dewey-Buchtitel) überhaupt abgebrochen werden musste. Der Wissenschaftshistoriker E. P. Fischer nennt sogar ein exaktes Datum für „die Zerstörung der alten Gewissheit“; es war der Zeitpunkt, zu dem die Wissenschaft sich genötigt sah, die Frage nach der wahren Natur des Lichtes als wissenschaftlich prinzipiell nicht eindeutig beantwortbar anzuerkennen (2005, 166, allg. $109 \mathrm{ff}$., $160 \mathrm{ff}$.). Gewissheit ist seither eine unwissenschaftliche Kategorie. Dewey hält sich lieber an die vertrautere Terminologie, wenn er den gemeinten Abbruch der „Suche nach Gewissheit“ mit der „kopernikanischen Wende“ in Verbindung bringt, wie sie erstmals Kant seinerzeit als „Analogie“-Formel erfolgreich eingeführt hat, um für die Pointe des mit seiner Erkenntnistheorie fälligen Paradigmenwechsels ein griffige Parallele aus der Wissenschaftsgeschichte $\mathrm{zu}$ nutzen $(1974,25)$. Eine „Analogie“ oder - so Blumenberg (1965, 100) - eine „Metapher“

43 Vgl. Rapp, Christof. Aristoteles zur Einführung. Hamburg: Junius, 2001, 122 ff., 131 ff.; Höffe, Otfried. Aristoteles. München: C.H. Beck, 1996, 32. 
war diese Kant'sche Formel und als solche hat sie auch historisch primär Erfolg gehabt, weil sie sich nicht für die faktischen Folgen interessierte, die mit dem Wechsel vom ptolemäischen zum kopernikanischen Weltbild für Theologie, Religion und Gesellschaft verbunden waren, sondern weil sie diese kosmologische Wende als lehrreiches Beispiel nutzte, um an ihm die Pointe einer vergleichbaren erkenntnistheoretischen Wende bzw. „Revolution der Denkart“ plausibilisieren zu können, wie sie Kant vorschlug (s. u. Kap. 8). Und diese Pointe meinte: Man versteht unser Erkenntnisvermögen (und seine Grenzen) besser, wenn man den von Kopernikus seinerzeit vorgenommenen Standpunktwechsel des Beobachters als ingeniösen Vorschlag nutzt, beim Erkennen das Erkenntnissubjekt sich nicht nach den Erkenntnisgegenständen, sondern umgekehrt die Erkenntnisgegenstände sich nach dem Erkenntnissubjekt und seinen Beobachtungs- bzw. Erkenntnisbedingungen richten zu lassen. Oder mit Deweys Worten von 1929 reformuliert: Nach der erkenntnistheoretisch adoptierten „kopernikanischen Wende“ „ist der Geist nicht länger ein Zuschauer, der die Welt von außen betrachtet und seine höchste Befriedigung im Genuss einer sich selbst genügenden Kontemplation findet. Der Geist ist in der Welt als ein Teil ihres voranschreitenden Prozesses“ (Dewey 1998, 291; vgl. Kuhn 1980). Dadurch wird aus einem vermeintlich objektivistischen „Erkennen als einem Betrachten von außen“ ein „Erkennen als aktive Teilnahme“ am Erkenntnisprozess und entsprechend an der Konstitution der Erkenntnisgegenstände selbst (Dewey 1998, 291), was „mit dem alten philosophischen Begriff von Wahrheit nichts mehr zu tun hat“ (Arendt 1960, 282-283 und bes. unten Kap. 8). Natürlich auch nichts mehr mit dem alten Versprechen, das z. B. Kopernikus' Wissenschaftsinteresse noch motivational antrieb, nämlich: dass eine Welt, die Gott für den Menschen (,propter nos“) als ihren privilegierten Zuschauer gemacht habe, auch vom Menschen in ihrer inneren Prozessdynamik (etwa was die Bewegungen der Himmelkörper angeht), verstehbar sein müsse (Blumenberg 1965). „Kränkung“, von der Freud später (im Plural) sprechen wird (s. Wetz 1994, 125 ff.), war also nicht erst die Reaktion auf eine systematische Enttäuschung dieser lange gehegten Verstehbarkeitsunterstellung, „Kränkung“ war historisch auch der vorgängige Impuls gegen das als „anstößig“ (so Kopernikus!) empfundene „Missverhältnis zwischen der metaphysischen Weltqualität und dem theoretischen Weltanblick, zwischen postulierter Weltordnung und praktischer Weltkonstruktion“ (Blumenberg 1965, 50). Doch die Arbeit an der Beseitigung dieser Kränkungsursache war bekanntlich nicht sehr erfolgreich: aus Wissenschaft als willkommener Gehilfin der Religion, die dem Glauben seine Vernünftigkeit attestieren und so als Weg „zu Gott“ fungieren sollte, wurde eine „gottfremde Macht“ (Weber 1967, 21), deren selbst methodologisch gezähmter Atheismus Gott in wissenschaftlichen Argumentationszusammenhängen keinen Platz mehr einräumen mochte, was absoluten Gewissheitschancen ebenso ihre 
Basis nahm wie das Glückversprechen kontemplativer Wahrheitszugänglichkeit („Wissenschaft als Weg zum Glück“ (Weber 1967, 21; vgl. Pieper 2012, 72-73)) verweigern musste.

Ist aber nur noch „die Gewissheit der Ungewissheit“ (Pörksen 2008) gewiss, dann ist damit auch explizit und endgültig ein Paradigma verabschiedet, das Dewey die „Zuschauertheorie des Erkennens [meine Hervorhebung, J. K.]“ nennt (1998, 27), die wir oben (Kap. 3) bereits gleichsinnig als jahrhundertelang geltendes Modell kontemplativer und d. h.: rezeptiver Erkenntnis- und Wahrheitsfindung skizziert haben. In ihm konnte noch als inhärente Eigenschaften der Dinge selbst ausgegeben werden, was wir heute „Konstrukte“ des Erkennenden zu nennen genötigt sind (vgl. dazu unten Kap. 8), insofern wir die Dinge auch kognitiv nur so erfassen, wie es für unser Überleben förderlich ist, weil unser Gehirn primär kein Organ des interesselosen Erkennens, sondern des Überlebens ist - was schon Nietzsche kühn behauptete (vgl. dazu Kopperschmidt 1994, 51-52) und heute neurobiologisch wie evolutionstheoretisch stützbar ist. Entsprechend dürfte - so Hawkings vergleichbares, nur forscheres Resümee in Der große Entwurf (2010) - heute kein Wissenschaftler mehr ernsthaft glauben, durch kontemplatives „Zuschauen“ statt durch gezieltes, in der Regel technisch unterstütztes experimentelles „Beobachten“ Erkenntnisse gewinnen zu können (die freilich vom Beobachten nicht unbeeinflusst bleiben); und nur notorisch Uninformierte dürften heute noch hoffen, dass sich wenigstens in den vermeintlich exakten modernen Naturwissenschaften Oasen stabiler Ordnung finden ließen, während wir doch längst wissen könnten, dass sowohl im Mikro- wie im Makrokosmos die gleiche Veränderungsdynamik herrscht wie auf unserer Erde. Diese Vorstellung, „dass unsere Welt unentwegt in Bewegung ist“ und es nirgends „absolute Ruhe“ „gibt“, dürfte den meisten Menschen freilich - so Fischer $(2005,150)$ - eher Angst machen denn von ihnen - wie noch bei Hawking im eben genannten Großen Entwurf (2010, 19 ff., 167 ff.) - als „großer Triumpf“ der menschlichen Erkenntnisfähigkeit gefeiert zu werden, weil sie endlich durch Einsicht in die „Gesetze der Natur“ die Unterwerfung unter den „Willen eines allmächtigen Gottes“ unnötig gemacht habe. Aus Rosas Bestseller über Beschleunigung (2005) als spezifische Zeitsignatur moderner Gesellschaften wissen wir freilich mittlerweile, wie sehr die als Beschleunigung gegenwärtig erfahrene (technische, geistige und soziale) Veränderungsdynamik bereits als Belastung und Überforderung empfunden wird, die eine Suche, wenn schon nicht nach Gewissheit, so doch wenigstens nach „Entschleunigung“ mehr als plausibel macht. ${ }^{44}$

44 Vgl. Fischer, Ernst Peter und Wiegandt, Klaus (Hgg.). Dimensionen der Zeit. Die Entschleunigung unseres Lebens. Frankfurt: Fischer, 2012. Zur folgenreichen „Gegenwartsschrumpfung“ durch Beschleunigung s. Lübbe, Hermann. Im Zuge der Zeit. Berlin und Heidelberg: Springer, 2003. 
Und was bedeutet das für Rhetorik? Sehr viel, meine ich! Man muss diese Andeutungen über den zeittypischen wie (vielleicht) endgültigen Verlust von Gewissheitschancen nur mit dem schon öfters erwähnten Blumenberg'schen Schlüsseltheorem in Verbindung bringen, um die Konsequenzen dieses Gewissheitsverlustes für unser Frageinteresse nach der philosophischen Relevanz der Rhetorik sofort zu erkennen: „Solange die Philosophie ewige Wahrheiten, endgültige Gewissheiten [!] wenigstens in Aussicht stellen mochte, musste ihr der consensus als Ideal der Rhetorik, Zustimmungen als das auf Widerruferlangte Resultat der Überredung, verächtlich erscheinen [meine Hervorhebung, J. K.]“ (1981, 112), will sagen: Solange man „die Suche nach Gewissheit“ noch mit gutem Gewissen betreiben konnte, musste der Rhetorik jede philosophische Dignität abgehen, weshalb die Philosophen auch mit Rhetorik wenig anzufangen wussten, außer sie brauchten sie als Nothilfe für ihr Überleben in der Höhle. Wenn jedoch nach dem bisher Gesagten das Blumenberg'sche „solange“ für die Gegenwart nicht mehr gelten kann, weil „der Philosophie der Verzicht [!] nicht erspart blieb, der aller [!] Rhetorik zugrunde liegt [meine Hervorhebung, J. K.]“, wenn also das „ontologische Vorurteil“ der Tradition(Riedel1972, 88) bzw. ihre „ontologischeSchlagseite“(Baecker 2005, 8) mit einer eindeutigen Präferenz für das Unveränderlich-Statische wissenschaftstheoretisch für uns bereits befremdlicher geworden ist als der modernitätsspezifischen Verzicht auf wissenschaftlich mögliche objektive Gewissheit überhaupt, - wenn das so ist, dann wird mit dem von Blumenberg eingeklagten „Verzicht“ auch die traditionell trennscharfe Grenze zwischen absolut sicherem Wissen bzw. Wahrheit und bloßen Meinungen nicht nur porös, sondern grundsätzlich aufgehoben zugunsten einer Unterscheidung zwischen wissenschaftlich geltenden oder herrschenden und strittigen Meinungen. Das aber muss fundamentale Konsequenzen für eine Rhetorik haben, deren „Reich“ (Perelman 1980) ja aus genau diesen Meinungen besteht, die keinen direkten Zugang zur Welt für sich reklamieren können, sondern „nur“ Geltungsansprüche für sich und ihre Aussagen über die Welt erheben, die bestritten, verteidigt, gestützt, eingelöst, akzeptiert usw. werden können. Jetzt und erst jetzt kann Rhetorik wirklich philosophisch interessant, ja relevant werden, weil ihr überzeugungsbedingtes Zustimmungsprinzip zu einem allgemeinen Geltungsprinzip avancieren kann, das nicht mehr nur - wie noch bei Aristoteles - im spezifischen Bereich von kontingenter Praxis das Fehlen „letzter Gewissheiten“ kompensieren soll, sondern in dem Maße seinen bisherigen Geltungsbereich sprengen kann, als die traditionellen Bestimmungen von Praxis verallgemeinert werden müssen zu Bestimmungen unseres Umgang mit Welt überhaupt. Der muss nämlich als ein durch und durch meinungshaft bzw. doxastisch geprägter Umgang verstanden werden (Rorty 1988, 16), der entsprechend auch nur Geltungsansprüche erheben kann, deren Anerkennung sich allein überzeugungsbedingter Zustimmungsnötigung verdankt 
(s. u. Kap. 8). Die Modernität einer solchen rhetorisch geprägten Geltungstheorie besteht nach Rorty darin, dass „Wahrheit“ nichts mehr ist, was - wie die Tradition wollte - „gefunden wird“ (etwa durch Kontemplation), sondern eher - wie die Sophistik wollte - was „gemacht wird“ (1991, 37, 96; 1987, 176).

Dieses Szenario einer geltungstheoretisch und damit philosophisch attraktiv gewordenen Rhetorik kann natürlich für Aristoteles noch nicht unterstellt werden. Dennoch! Auch bei Aristoteles ist neben der Konzessionsrhetorik und der aus ontologischen Gründen als bloßer Mängelkompensation im Bereich von Praxis funktionalisierten Verlegenheitsrhetorik schon von einer anderen Rhetorik zumindest ansatzweise die Rede, über die in den bisherigen zwei Argumenten für die Nützlichkeit der Rhetorik noch gar nicht gesprochen worden ist. Doch Aristoteles kennt wie oben gesagt - insgesamt mindestens vier solcher Nützlichkeitsargumente (vgl. auch Rapp 2002/1, 79 ff.), von denen die beiden restlichen (c und d) durchaus geeignet wären, die bisherige Funktionsbestimmung der Rhetorik ( $\mathrm{a}$ und b) fast vergessen zu machen; denn diese andere Rhetorik ist in der Tat alles andere ist als „ein bloßes Resignationsideal“. Sie darf nicht einmal mit der Idee einer positivierten Rhetorik verwechselt werden, die Aristoteles nach Heideggers tollkühner These bei Platon entdeckt haben und in seiner „Rhetorik“ operativ ausgebaut haben soll, während Aristoteles in Wahrheit mit seiner „Rhetorik“ sich wie kein anderer von Platon und dessen Idee von Rhetorik konzeptionell entfernt hat. Zwar gilt nach wie vor noch - auch für Aristoteles - die von Riedel eingeklagte fatale „Aporie“ als notwendiger Folge des o. g. „ontologischen Vorurteils“ der Tradition, dass es nämlich „,von Gegenständen, die veränderlich und unselbständig sind und somit auch Veränderung durch Handeln zulassen, kein methodisch gesichertes Wissen gibt, dass jene Gegenstände hingegen, die ein solches Wissen zulassen, infolge ihrer Beschaffenheit [der Unveränderlichkeit bzw. Selbstständigkeit] nicht zu verändern sind“ (1972, 88-89). Doch selbst wenn man die prekäre Wirkung dieser „Aporie“ auf die europäische Wissenschaftsgeschichte und besonderes auf die praktische Philosophie nicht unterschätzen darf, sie hat die Suche nach einem Verfahren nicht lähmen können, das die „Veränderung durch Handeln“ auch ohne „gesichertes Wissen“ zu methodisieren sich zutraute (vgl. Buchheim 1986, 77 ff.). Aristoteles hat diese mögliche Methodisierungsleistung der Rhetorik zugetraut und für diese rhetorische Leistung sogar einen Schlüsselbegriff geprägt, der längst in lateinsicher Übersetzung in die einschlägige Reflexionsgeschichte eingegangen ist und bis heute in einer emphatischen Weise gebraucht wird, die kaum nach ahnen lässt, dass es sich einmal um eine ontologisch bedingte Mängelkompensation gehandelt hat; gemeint ist der Begriff „Deliberation“. ${ }^{45}$

45 Vgl. Buchheim 2000, 113 ff.; Habermas 1992, 349 ff. und unten Kap. 12. 
Dieser latinisierte Übersetzungsbegriff für den griechischen Originalbegriff bouleusis (Beratung) ist aus zwei Gründen als Schlüsselbergriff für unser Frageinteresse geeignet: Einmal fungiert er bei Aristoteles als Brückenbegriff, der die oben bereits erläuterte Beziehung zwischen der Logizität und Polizität des Menschen operationalisiert, insofern sich in „Deliberation“ das Sprachlich-Werden bzw. das Zur-Sprache-Kommen von Politik operativ vollzieht. Zum anderen bietet der Begriff „Deliberation“ die willkommene Chance, als Anschlussbegriff zu fungieren, mit dessen Hilfe sich unser Frageinteresse an eine internationale Diskussion andocken lässt, die unter dem Namen „deliberative Politik“ geführt wird und in deren Rahmen Habermas einen Begriff von „kommunikativer Vernunft“ konturiert hat (1985a, 344 ff.), der für jedes Rhetorikkonzept, das Vernunft zum Sprechen bringen will, attraktiv werden muss. Das u. a. auch deshalb, weil die Spezifikation „kommunikativ“ Vernunft nicht „material“ bzw. „substanziell“, sondern „formal“ bzw. „prozedural“ bestimmt und damit die Idee von Vernunft als „Verfahrensrationalität“ entwirft (Habermas 1988, 42 ff.). Dadurch kommt sie einer Theorie entgegen, die wie die Rhetorik auch über keine eigenen materialen Überzeugungsressourcen verfügt, sondern nur ein Verfahren zu methodisieren verspricht, mit dem jeweils unterstellbare Überzeugungsressourcen problembezogen und adressatenspezifisch aktualisiert werden können, um erfolgreiche Problemlösungen zu finden. Als erfolgreich dürften aber heute, wie einschlägige aktuelle Beispiele leicht belegen könnten, weithin nur noch Problemlösungen gelten, die durch überzeugungsbedingte Zustimmungen gedeckt sind und damit den fälligen sozialen Kooperationsbedarf moderner Gesellschaften nur noch aus zustimmungsbezogener Verständigungsarbeit $\mathrm{zu}$ befriedigen vermögen.

\subsection{Umrisse einer anderen Rhetorik}

Der Begriff „Deliberation“ (verständigungsbezogene Beratung) spezifiziert bei Aristoteles nicht mehr bloß eine der drei traditionellen Redegattungen (genus deliberativum), sondern charakterisiert die spezifische Leistung einer funktionalen Sprachverwendung überhaupt, wie sie die Rhetorik methodisiert (vgl. Kopperschmidt 1995, 88 ff.). Zum Schlüsselbegriff avanciert „Deliberation“ bei Aristoteles, weil sie ein Verfahren kennzeichnet, das zwar fehlende Evidenzen von Praxis kompensieren muss (,Wir beraten nur über Dinge“, über die wir „keine Gewissheit besitzen können“, Rhet. 1357a; vgl. NE 1130b), zugleich aber mit diesem Kompensationszwang ein Niveau möglicher Kooperation erschließt, das Praxis qualitativ völlig zu verändern in der Lage ist. Denn was Deliberation operativ ermöglicht, ist Kooperation mittels einer auf zustimmungsbezogener Verständigung basierenden und durch sie motivierten Handlungskoordination, die 
ihrerseits Subjekte voraussetzt, die fähig und bereit sind, die „Möglichkeit des Anders-Seins“ positiv als Handlungsoptionen bzw. als Entscheidungsoptionen zu nutzen und sie nach theoretischen wie praktisch/normativen Kriterien zu selektieren, die von den Handelnden selbst verantwortet werden. Was also ontologisch durchaus ein Mangel sein und bleiben mag, kann handlungstheoretisch zur Bedingung möglicher (praktischer) Optionalität und Freiheit werden (vgl. Riedel 1972, 88), sodass man wegen dieser Folgen sogar - in Anlehnung an eine vergleichbar paradoxe Formel ${ }^{46}$ - von einem vorteilhaften Mangel (felix inopia) reden könnte, über den sich Mängelkompensations- und Verständigungsparadigma miteinander versöhnen lassen. Das sagt Aristoteles zwar so nicht und meint es auch nicht so, und doch ist das ontologische Defizit von Praxis im Sinne von „Möglichkeit des Anders-Seins“ auch für ihn zugleich die notwendige Voraussetzung der Möglichkeit von Politik (und veränderungsbezogener Praxis überhaupt), die ihrerseits wieder als Voraussetzung eines als spezifisch menschlich definierten Lebens (zoon politikon) fungiert, das nach dem oben Gesagten nur eine durch Sprache vermittelte Kooperation zwischen „Freien und Gleichen“ (Polit. 1255b und öfter; Schütrumpf 1980, 74 ff.) sein kann. So lautet nämlich Aristoteles’ griffige Formel für diese „höchste“ und allein als politisch qualifizierte „Herrschaftsform“ (arche) (Polit. 1252a), die bis heute (meist zitatfrei, besonders bei Habermas) für das Programm und Ideal demokratischer Selbstbestimmung steht. Dass für Aristoteles die Bedingungen solcher Selbstbestimmung von „Freien und Gleichen“ seinerzeit natürlich nur eine männliche Minderheit erfüllen konnte, muss den Wahrheitsgehalt dieses folgenreichen Ideals nicht mindern, allenfalls den uneingeschränkten Geltungssinn dieses Ideals einklagen. Deliberative Verständigung unter „Freien und Gleichen“ bleibt das attraktive Leitbild für eine politische Existenz, die nicht bloß ein „Überleben“ sichern will (wie bei Hobbes) noch bloß im Kampf um wirksamere Herrschaftstechniken sich erschöpft (wie bei Machiavelli), sondern ein „glückhaft gelingendes Leben“ intendiert (Polit. 1252a) - und zwar im Hier und Jetzt. Rhetorik, wenn man sie mit Aristoteles als Theorie, Praxis und Methodisierung solcher Kooperation ermöglichenden Deliberation versteht, ist dann aber auch keine bloße „Verlegenheits-“ oder Mängelkompensationstheorie mehr und erst recht natürlich keine bloße Konzessionstheorie, sondern sie ist theoriestrategisch längst auf dem (freilich noch langen) Weg zu ihrer Selbstdefinition als deliberativer Verständigungstheorie für Freie und Gleiche.

Zwei der insgesamt vier Nützlichkeitsgründe aus Aristoteles' Rhetorik sind wie gesagt - bisher noch gar nicht herangezogen, die aber für die eben genannte

46 „felix culpa“ bezeichnet seit Augustin die Ursünde als „glückhafte Schuld“ des Menschen, weil sie zur Menschwerdung des Erlösers geführt hat; in der katholischen Osternacht-Liturgie ist dieses Oxymoron bis heute präsent. Vgl. dazu allgemein Marquard 1995, $11 \mathrm{ff}$. 
evolutionäre Einschätzung der Aristotelischen Rhetorik und ihre Ansätze einer „anderen Rhetorik“ fundamental sind. Das soll jetzt endlich nachgeholt werden. Der eine Grund lässt sich als weitere

c) Nützlichkeitsbestimmung aus der Aristotelischen Politik entnehmen, die an einer für unser Frageinteresse wichtigen Stelle ganz un-, ja antiplatonisch dafür plädiert, das eben genannte Potential deliberativer Verständigung durch systematische Entgrenzung der Zugangsbedingungen zu dieser Verständigungsarbeit zu vergrößern (Pol. II 2 ff.; vgl. Kopperschmidt 1995, 85 ff.). Dass hier nicht mehr bloß von Kompensationsleistungen ontologischer Defizite die Rede ist, sondern weit mehr vom originären, nämlich summativen Leistungsgewinn kollektiver Verständigungsarbeit, lehrt die erkennbare Nähe dieser „Summierungstheorie“ (vgl. Rapp 2002/2, 130; Ottmann 2005, 214) zu modernen (sozialpsychologischen) Theorien über den „Vorteil der Gruppe“ oder Die Weisheit der Vielen (Surowiecki 2005), was neuerdings gern „Schwarmintelligenz“ genannt wird (s. Miller 2010), oder erst recht zu Hannah Arendts Apologie der Pluralität (2016). Damit wird Aristoteles' Theorem sogar evolutionsgeschichtlich anschlussfähig, insofern es die Klugheit der „Vielen“ (sofern sie nur „freie Männer“ (!) sind und „sich an Gesetze halten“) der vermeintlichen Weisheit der Wenigen (auch der monologisierenden „Philosophenkönige“!) als überlegen einschätzt (s. u. Kap. 8). Noch wichtiger erscheint mir, dass mit der summierungstheoretischen bzw. kognitiven Aufwertung der „Vielen“ (plethos/hoi polloi) durch Aristoteles nicht nur ihrer notorischen Verachtung durch Plato widersprochen wird, sondern dass sich positive Anschlusschancen an bedeutende und - wie im Fall von Perelman etwa - sogar an rhetorikaffine Theorien ergeben, die den Grad der Öffentlichkeit zum Maßstab für die Qualität und Vernünftigkeit deliberativer Verständigung machen (s.u. Kap. 6). ${ }^{47}$ Habermas meint sogar, dass man die oben erläuterte Aristotelische These, wonach der Mensch ein zoon politikon sei, erst recht verstehe, wenn man „politikon“ mit „im öffentlichen Raum existierend“ übersetze $(2005,17)$. Dem ist vorbehaltlos zuzustimmen, zumal damit implizit noch einmal die oben behauptete enge Beziehung zwischen Polizität und Logizität (in der Doppelbedeutung von sprach- und vernunftfähig) des Menschen bestätigt wird: Der Mensch muss nach Aristoteles „ein redendes Wesen“ sein, um „ein im öffentlichen Raum existierendes“ und seine Vernunft so zur Geltung bringendes Wesen sein zu können. Im bereits zitierten

47 Aristoteles erinnert in diesem Zusammenhang an eine allgemeine Erfahrung, dass nämlich „Menschen nicht ein und dasselbe öffentlich und privat loben, sondern öffentlich nur das Gerechte und Treffliche, während sie privat eher den eigenen Vorteil anstreben“ (Rhet. 1399a). Interessant ist diese Erfahrung als Hinweis auf ein bis heute (s. auch soziale Netzwerke) nachweisbares höheres intellektuelles und moralisches Niveau öffentlichen Redens, das sich für politische Entscheidungsprozesse nutzen lässt (vgl. Pol.1281). 
Titel der Habermas-Festschrift Die Öffentlichkeit der Vernunft und die Vernunft der Öffentlichkeit (2001) findet nicht nur diese dialektische Denkfigur in einer entsprechenden Stilfigur („Antimetabole“) ihren sprachlich angemessenen Ausdruck, sondern auch das Programm einer Rhetorik findet in ihr eine pointierte Verdichtung, die den öffentlichen Vernunftgebrauch im Interesse kooperativer Handlungsermöglichung für „freie und gleiche“ Subjekte methodisieren will. Selbst wenn Reden-Können dem Menschen nach Aristoteles zur Ermöglichung seiner politischen Existenzweise dient (s. o. Kap. 4.1), so ist damit die ganze Funktionsbreite von Rede natürlich genau so wenig benannt wie die politische Existenz die einzige Weise menschlichen Lebens beschreibt; doch nobilitiert sie eine Existenzweise, in der das Reden-Können seine vornehmste Funktion gewinnt, insofern es - so Nietzsches klassische Formulierung (KGA II 4, 113ff.) - zum „größten Machtmittel inter pares [meine Hervorhebung, J. K.]“ zu werden vermag, d. h.: zum „größten [ich würde lieber sagen: zum einzigen] Machtmittel“ unter gleichberechtigten Subjekten in ihrer Rolle als Bürger. Diese Bürgergemeinschaft aber ist keine bloß vergrößerte Hausgemeinschaft (bzw. Familie), sondern eine singuläre Assoziation „freier und gleicher“ Männer zum Zwecke der deliberativen Verständigung über die Praxis gelingenden Lebens, das mehr meint als „bloßes Überleben“ (Pol. 1280a). Deshalb ist für Aristoteles die strikte Unterscheidung zwischen „oikos“ und „polis“, zwischen „Haus“ und „Staat“ so konstitutiv (vgl. Ottmann 2005, 12-13), sodass, wer den politischen Raum betreten will, das Haus als herrschaftlich organisiertes Abhängigkeitsgefüge ${ }^{48}$ verlassen muss; denn nur im politischen Raum herrscht das Prinzip der „Pluralität“ (Arendt 1993, 9; 2016) und gilt entsprechend das Prinzip der Vielfalt möglicher Meinungen, die sich nicht nach Hausherrenart zur Einheit zwingen oder - so Platons Konzept (s. u. Kap. 7) durch Expertenwissen (Expertokratie) einfach ausschalten lassen, sondern die durch Überzeugungsarbeit um mehrheitsfähige Zustimmung werben müssen. „Macht“ bestimmt sich unter solchen Bedingungen nicht als erfolgreiche „Instrumentalisierung eines fremden Willens für eigene Zwecke“, sondern als gelingende „Formierung eines gemeinsamen Willens in einer auf Verständigung gerichteten Kommunikation“. So beschreibt Habermas sein „kommunikatives Handlungsmodell“, das explizit an Hannah Arendt und ihre kategoriale Unterscheidung zwischen „[sprachimprägnierter] Macht und [stummer] Gewalt“ (1975, bes. Kap. II) anschließt und darüber vermittelt natürlich auch an Aristoteles, dessen „Praxis“Begriff Arendt nach Habermas besonders in ihrem Hauptwerk Vita activa oder vom tätigen Leben (1960, bes. Kap. II) „systematisch erneuert hat“ durch eine

48 Vgl. zu dieser herrschaftlich durchorganisierten Hausgemeinschaft von Sklaven, Frauen und Kindern Pol. 1253b. 
gegen Platon eingeklagte kategoriale Abgrenzung der „Praxis“ von „Poiesis/ Herstellen“ und „Arbeit“(Habermas 1978, 103 ff.). Wenn nur die öffentliche „Mobilisierung von Zustimmung Macht erzeugt“ $(1978,104)$, wenn also „Macht sich nur im kommunikativen Handeln bildet“ und insofern „ein Gruppeneffekt der Rede ist“, dann wird Politik als genuiner „Praxis“-Raum zum genuinen Rede-Raum, ${ }^{49}$ in dem jener „eigentümlich zwanglose Zwang“ zur Geltung kommen kann, der gelingender Überzeugung eigen ist und deren „Vernunftanspruch“ selbst „erfolgreiche Manipulation“ von Überzeugungsarbeit nicht zu dementieren vermag, ${ }^{50}$ weil ja auch deren Erfolg sich nur dem erfolgreich prätendierten „zwanglosen“ Überzeugungszwang verdankt (1978, 106 und unten Kap. 11.3). Nach einem großartigen Wort von Arendt gibt es ,auf die Frage nach dem Sinn von Politik“ nur eine mögliche Antwort: „Der Sinn von Politik ist Freiheit“ (1993, 28, 35 ff.). Wenn diese Freiheit im Rede-Raum öffentlicher Überzeugungsarbeit unter „Freien und Gleichen" konkrete Praxis wird, dann avanciert Rhetorik erkennbar aus einer legitimen Strategie „wohlmeinender Lüge“ und Überredung bei Plato zu einem Medium operativer Ermöglichung von Freiheit (s. u. Kap. 11.3).

Dass Aristoteles in der Vorgeschichte eines solchen Rhetorikverständnisses einen prominenten Platz beanspruchen darf, bezeugt schließlich besonders

d) sein letztes und viertes Nützlichkeitsargument, das deshalb auch besondere Beachtung verdient. Denn an der gemeinten Stelle der Rhetorik formuliert Aristoteles eine These, mit der nicht nur Heidegger in seiner o. g. Vorlesung von 1924 nichts anzufangen wusste, obwohl ihr substanzieller Aussagegehalt für Ohren von Rhetorikern eigentlich (vergleichsweise) ungeheuerlich klingen müsste. In ihm wird nämlich nicht weniger als eine intrinsische und nicht bloß additive Beziehung zwischen Wahrheit und Überzeugungskraft behauptet, was die missliche Arbeitsteilung zwischen Philosophie und Rhetorik aufkündbar macht: „Die Rhetorik ist nützlich, weil das Wahre und Gerechte immer [!] [bzw. von Natur aus] größere Überzeugungskraft [pithanotera] besitzen als das jeweilige Gegenteil [meine Hervorhebung, J. K.]“ (Rhet. 1355a21 bzw. a37). Sollte sich dieses Konvergenztheorem plausibilisieren lassen, dann impliziert es erkennbar eine folgenreiche Konsequenz für die Rhetorik, insofern dann nämlich die mögliche

49 „Wortloses Handeln gibt es streng genommen überhaupt nicht“ (Arendt 1960, 168; allg. dazu 1994, 164 ff.). „Politisch zu sein [...], das hieß, dass alle Angelegenheiten vermittels der Worte, die überzeugen können, geregelt werden und nicht durch Gewalt oder Zwang“ (1994, 30).

50 Die These, dass auch „noch die erfolgreiche Manipulation Vernunftansprüchen Rechnung tragen muss“, soll natürlich nicht die Nürnberger Parteitage der NSDAP zu deliberativen Rede-Räumen verharmlosen, sondern nur die nicht tilgbare „Ambivalenz“ betonen zwischen „Überzeugung und Überredung, die dem rhetorisch hervorgebrachten Konsensus anhängt“ (Habermas 1973, 267); vgl. Kopperschmidt 2003, 181 ff., 327 ff. 
(graduell durchaus differenzierbare) Überzeugungskraft einer Aussage als ein (entsprechend differenzierbares) Indiz ihrer Wahrheit gelten müsste und die rhetorische Arbeit an der Überzeugungskraft einer Aussage würde zugleich Arbeit an der Erschließung ihrer möglichen Wahrheitsindikation sein. Dass sich hier eine höchst interessante Perspektive für die philosophische Rettung der Rhetorik jenseits ihrer bloß konzessiven oder verlegenen Duldung auftut, ist offenkundig, muss aber noch etwas genauer erläutert werden, als es der obige Satz tut. Denn der behauptet zwar einen intrinsischen, genauerhin: indikativen Zusammenhang zwischen der jeweiligen Überzeugungskraft einer Aussage und ihrer Wahrheit und begründet aus diesem Zusammenhang auch die Nützlichkeit der Rhetorik, doch lässt er völlig offen, worin diese Nützlichkeit operativ bestehen soll bzw. wie sich die Rhetorik mit Bezug auf diesen Zusammenhang denn nützlich machen könnte, wenn die o. g. traditionelle Arbeitsteilung zwischen Philosophie (Wahrheit) und Rhetorik (Wirkung) nicht mehr haltbar ist. Etwas später freilich folgt dann ein Satz, der argumentativ eigentlich schon hier fällig gewesen wäre, nämlich: dass es die originäre Aufgabe der Rhetoriktheorie sei, „zu erkennen, welche Ressourcen möglicher Überzeugungskraft [pithana] in einer jeweiligen Problemlage verborgen liegen“ (Rhet. 1355b). Fügt man diesen Satz argumentativ in den fraglichen Zitat-Text ein, dann erschließt sich der in ihm behauptete Nützlichkeitswert der Rhetorik fast von selbst - jedenfalls für eine Wahrheitstheorie, der die Überzeugungskraft von Rede als Wahrheitsindiz gilt: Rhetorik ist demnach nützlich, weil sie die Überzeugungskraft, die dem Wahren und Gerechten immer bzw. von Natur aus eher zukommt als dem Unwahren und Ungerechten, methodisch freilegen zu können verspricht und Überzeugungskraft damit als Wahrheitsindiz überhaupt erst operativ wirksam werden lässt.

Die Kühnheit dieses für den evolutionären Charakter der Aristotelischen Rhetorik grundlegenden Theorems dürfte damit präzis bestimmbar sein: Rhetorik ist nicht nur deshalb nützlich, weil sie das (vermeintlich) objektiv Wahre und Gerechte noch zusätzlich (additiv) überzeugungskräftig und damit für ,die Vielen“ leichter zustimmungsfähig zu machen versteht, sondern Rhetorik ist primär deshalb nützlich, weil sie die Überzeugungskraft als immanente, wenn auch für viele meist latente Eigenschaft des Wahren und Gerechten systematisch zur Selbstexplikation nötigt und so deren Zustimmungsfähigkeit faktisch stärkt. Denn je mehr Menschen sich an der Explikation bzw. Freilegung dieser überzeugungsgestützten Zustimmungsfähigkeit deliberativ beteiligen, desto größer muss nach summationstheoretischem Kalkül die Chance ihres Gelingens sein (s. o. unter c). Deshalb favorisiert Aristoteles - wieder anders als Platon - auch nicht mehr die monologische Gewissheitssuche, sondern eine möglichst entgrenzte Chance deliberativer Beteiligung. Rhetorik wird dadurch - so Rapps treffende Metapher (2002/2, 133, 85 ff.) - „eine Art Selbsthilfeeinrichtung des logos“, 
insofern sie das „natürliche [!] Überzeugungspotential“ des Wahren und Gerechten auffindbar zu machen hilft (vgl. auch Figal 1996). Entsprechend wäre es auch „schändlich, wenn sich die Rede [logos] nicht selber mithilfe der Rhetorik zu helfen wüsste, obwohl der Gebrauch der Rede doch weit eigentümlicher für den Menschen ist als der des Körpers“.

Doch worauf beruht eigentlich dieses „,natürliche Überzeugungspotential“ des Wahren und Gerechten bzw. wie begründet Aristoteles eigentlich die bisher nur behauptete intrinsische Beziehung zwischen dem Wahren/Gerechten und deren vergleichsweise größerer Überzeugungskraft, die es methodisch zu entbinden gilt? Es gibt, soweit ich sehe, keine direkte Antwort von Aristoteles auf diese Frage; wohl aber lässt sich eine indirekte Antwort finden, wenn man nämlich einschlägige Theoriestücke Aristotelischen Philosophierens zusammenträgt und in einen argumentativ konsistenten Zusammenhang bringt. Eben das will ich versuchen, indem ich von der These ausgehe, die dem Theorem von der Überzeugungskraft als immanenter Eigenschaft des Wahren und Gerechten unmittelbar vorausgeht. Es heißt dort: „Die Menschen [also nicht nur wenige Experten!] sind für das Wahre von Natur aus [!] hinlänglich begabt, und meistens treffen sie auch das Wahre; die anerkannten Meinungen [ta endoxa] zu treffen verlangt nämlich [!] die gleiche Begabung wie die Wahrheit zu treffen“ (1355a). Wer heute den ersten Teil dieser Behauptung mit Aussicht auf Zustimmung begründen wollte, würde schwerlich noch den Weg wählen können, den Aristoteles seinerzeit bedenkenlos gehen konnte; denn der Aristotelische Satz, der sich für die starke Behauptung einer natürlichen bzw. prinzipiellen Wahrheitsfähigkeit des Menschen (vgl. Rapp 2002/2, $72 \mathrm{ff}$.) als plausible Stützung anbietet, ist der Eröffnungssatz der Aristotelischen Metaphysik, was ihm ein besonderes Renommee verschafft: „Alle Menschen streben von Natur aus [!] nach Wissen“ (980a). Um als Begründung der prinzipiellen Wahrheitsfähigkeit des Menschen beansprucht werden zu können, fehlt eigentlich nur noch ein Theorem, das oben auch bereits zitiert und als Argument funktionalisiert worden ist, nämlich: dass „die Natur nichts umsonst tut“. Damit dürften alle relevanten Teilelemente eines konsistenten Begründungszusammenhangs komplett sein: Wie mithilfe des Arguments, dass die Natur nichts umsonst tue, oben die „natürliche“ Polizität des Menschen aus seiner Logizität begründet worden ist, so wird hier analog die Wahrheitsfähigkeit des Menschen mit seinem „natürlichen“ Wahrheitsstreben begründet; denn ebenso wie es sinnwidrig wäre, den Menschen mit einer singulären Sprachfähigkeit auszustatten, ohne mit dieser Sprachfähigkeit eine ihm allein eigene, nämlich „politischen“ Existenzweise ermöglichen zu wollen, ebenso wäre es sinnwidrig, ihn mit einem „natürlichen“ Wahrheitsstreben auszustatten, ohne diesem Wahrheitsstreben je eine Befriedigungschance zu gönnen. In beiden Fällen hängt erkennbar die Plausibilität der Argumentation definitiv von einer ihr zugrundeliegenden teleologischen bzw. 
finalistischen Denkfigur ab, deren Akzeptabilität sich der Weigerung verdankt, die Natur könne sich - so die beliebte einschlägige Metaphorik - wie eine „,böse Stiefmutter“ (noverca) verhalten. ${ }^{51}$ Nur wenn diese Weigerung allgemein unterstellbar ist, kann ein teleologischer Argumentationszusammenhang wie der hier rekonstruierte wirksam werden. Das gilt nach Blumenberg ebenso für das von ihm zitierte sinnverwandte Aristoteles-Theorem, dass ,wir das, wovon alle überzeugt sind, ,wirklich“ nennen“ (NE 1172b): Auch für die Plausibilisierung dieser These muss man - so Blumenberg - ,immer schon ein teleologisches Argument im Hintergrund [haben]“ (1981, 108), das ein Misslingen überzeugungsgeleiteter Wahrheitssuche weithin auszuschließen hilft; deshalb darf auch die oben bereits zitierte Annahme gelten, dass sich das „Treffen anerkannter [weil überzeugungskräftiger] Meinungen“ und das „Treffen der Wahrheit“ der gleichen Kompetenz verdankt (Rhet.1355a), sodass sich das Treffen überzeugungskräftiger Meinungen - ähnlich wie der „consensus omnium“ (allgemeine Übereinstimmung) ${ }^{52}$ - als verlässliches Indiz dafür nutzen lässt, dass man sich bei der Wahrheitssuche auf der richtigen Spur befindet. „Erst die skeptische Zerstörung [meine Hervorhebung, J. K.]“ dieser teleologisch gesicherten Annahme - und diese „skeptische Zerstörung“ ist nicht nur für Blumenberg heute natürlich schlichte Faktizität $t^{53}$ - macht die Frage dringlich, ob und wie sich Überzeugungskraft als immanenter Eigenschaft von Wahrheit auch ohne teleologischen „Rückhalt“ retten ließe. Gerettet aber muss diese intrinsische Beziehung zwischen der Wahrheit einer Rede (im vorerst verallgemeinerten Sinn theoretischer wie praktischer Gültigkeit, s. u. Kap. 10.2.2) und ihrer Überzeugungskraft, weil von dieser Beziehung definitiv das Gelingen jeder philosophischen Rettung der Rhetorik abhängt, da Rhetorik es nun einmal „positiv oder negativ [immer] mit der Wahrheit zu tun hat [meine Hervorhebung, J. K.]“ (Blumenberg 1981, 105). Wie also ließe sich diese intrinsische Beziehung ohne teleologische Stütze retten?

51 Vgl. zum „noverca“-Theorem usw. unten Kap. 5.4! „Natur“ ist natürlich ein Pseudonym bzw. Metonym für ein gottähnliches Prinzip.

52 Vgl. Suhr, Martin. Artikel „,consensus omnium, consensus gentium“. HWPh, Bd. 1. Hgg. Ritter, Joachim et al. Basel: Schwabe Verlag, 1971, $1031 \mathrm{ff}$.

53 An der heutigen Unbekanntheit des 1955 bzw. (in Deutschland) 1959 erstmals erschienenen und seinerzeit engagiert diskutierten Buches Der Mensch im Kosmos (München: C.H. Beck) von Pierre Teilhard de Chardin lässt sich die Fremdheit teleologischer Denkstrukturen in der Wissenschaft beispielhaft ablesen, die Richard Dawkins in Der blinde Uhrmacher (München: Kindler, 1986 bzw. München: dtv, 1990) destruiert hat, indem er die seit William Paley (1802) mit der Uhrmacher-Metapher abgebildete und göttlich begründete Finalität der Welt durch zwei Prinzipien der Evolutionstheorie (Mutation und kumulative Selektion) wissenschaftlich erfolgreich zu ersetzen versucht. 


\subsection{Zwischen Geltungsindiz und Geltungskonstitution}

Von Blumenberg kann man sich an eine zunächst äußerst gleichsinnig klingende Formulierung vis veritatis (Kraft der Wahrheit) erinnern lassen, die der christliche Rhetor Laktanz (viertes Jahrhundert) prägte, um mit ihr eine der göttlichen Wahrheit selbst inhärente Überzeugungskraft zu bezeichnen, die auch und sogar der nuda veritas, d.h.: der von allem rhetorischen Schmuck entblößten Wahrheit anhaften und ihr einen eigensinnigen Schmuck (satis ornata per se) verleihen soll, der jeden, der sie verteidigt, eloquent macht (diserte copioseque defendere veritatem) (Blumenberg 1998a, 59). Diese paradoxe Rhetorizität rhetorikfreier Wahrheit eine der „List der Vernunft“ (Hegel) vergleichbare „List der Wahrheit" nennen zu wollen, verbietet sich angesichts des göttlichen Charakters dieser Wahrheit; gleichwohl stellt dieses Paradox eine „perfekte Unionsformel“ für „das Verhältnis von Wahrheit und Rhetorik“ dar (1998a, 57): Wenn die Wahrheit nämlich eine ihr eigene Überzeugungskraft (vis) besitzt, dann muss diese auch nicht mehr zum bloßen „Instrument der Wahrheit“ sozialtechnologisch degradiert werden, sondern kann zu deren „Ausdruck“ (!) avancieren: Dann „ist der Glanz der Diktion der Glanz der Wahrheit selbst, unmittelbare Selbstumsetzung der Sache in die Sprache und ihre Überzeugungskraft“ (1998a, 59). So attraktiv sich diese Beschreibung Blumenbergs auch gibt und so sehr sie auch überzeugungspsychologisch recht haben mag, insofern die größte Überzeugungsressource fraglos das Überzeugtsein des Redners von der Wahrheit seiner Aussage ist, ein entsprechendes Rezeptionsorgan für die rhetorikfreie Rhetorizität göttlicher Wahrheiten würde ich mit Augustin dennoch nur bei ganz wenigen unterstellen wollen, weshalb seine pragmatische Rettung der Rhetorik als einer zusätzlichen Zustimmungsstimulanz wohl auch erfolgreicher war. Wer freilich wie Wilfried Stroh der Auffassung ist, dass ,die Stiftungsurkunde einer christlichen Rhetorik" bei Laktanz zu finden sei, muss nach Belegen dafür suchen, dass die Augustinische Pragmatik auch Laktanz nicht ganz fremd war und er gelegentlich - so der gefundene Beleg - nicht nur der „eigenen Kraft der Wahrheit“ vertraute, sondern auch auf „den Zauber der Rede“ setzte, um der Wahrheit zum Erfolg zu verhelfen (Stroh 2009, 499). Ob solche Belege ausreichen, um als „Stiftungsurkunde einer christlichen Rhetorik" gelesen werden zu dürfen, mag dahin gestellt bleiben; ich würde diesen Ehrentitel eher Augustins De doctrina christiana vorbehalten, weil ich überzeugt bin, dass dieses Werk weit mehr bietet als das, was Stroh in ihm sieht, nämlich bloß „eine christliche Predigtlehre“ $(2009,502)$. Wichtiger aber für unseren Fragekontext ist, dass beide christlichen Rhetoriker einen Erklärungsgrund für eine wahrheitsinhärente Überzeugungskraft bereithalten, der seinerseits wieder Prämissen - diesmal theologische - voraussetzt, die heute genauso chancenlos sein dürften wie die von Aristoteles unterstellten 
teleologischen, was deren Akzeptabilität angeht. Gibt es jenseits dieser obsoleten Prämissen wirklich keinen plausibleren Grund für die Überzeugungskraft als wahrheitsinhärenter Eigenschaft und damit für die philosophische Rettung der Rhetorik?

Doch, es gibt ihn! Er ist in dem Blumenberg-Satz bereits implizit genannt, der oben schon teilweise zitiert wurde, als von der „skeptischen Zerstörung des Rückhalts“ die Rede war, der traditionell die intrinsische Beziehung zwischen Wahrheit und Überzeugungskraft noch teleologisch abzusichern vermochte. Und dann folgt ein oben ausgesparter, hier aber dringend benötigter Satzteil, der benennt, was diese skeptische Rückhalt-Zerstörung positiv geleistet hat: Sie hat nach Blumenberg ,den pragmatischen Untergrund wieder sichtbar gemacht“, auf dem die gemeinte Beziehung zwischen Wahrheit und Überzeugungskraft sich skepsissicher fundieren ließe. Dieser ,pragmatische Untergrund“ heißt consensus bzw. Übereinstimmung aufgrund überzeugter Zustimmungsnötigung (1981, 108-109). Damit ist gemeint: Wahrheit ist nicht deshalb vergleichsweise so überzeugungsstark, damit sie auf breite Zustimmung stoßen und sich so durchsetzen kann, sondern Wahrheit ist so überzeugungskräftig, weil ihre Überzeugungskraft selbst bereits vorgängiger Grund und vorgängiges Kriterium für ihre Anerkennung als wahrheitsfähiger Geltungsanspruch ist. Überzeugungskraft ist mithin keine Folge von Wahrheit, sondern deren Geltungsbedingung. Damit verändert sich aber die Funktion überzeugter Zustimmungsnötigung, insofern aus ihrer Wahrheit bloß indizierenden eine Wahrheit überhaupt erst konstituierende Leistung wird. Wahrheit, die sich solchermaßen als zustimmungsabhängiger Geltungsanspruch versteht, kann dann natürlich keine mögliche Eigenschaft von Dingen in der Welt mehr meinen, also keine ontologische Kategorie mehr sein, sondern Wahrheit wird zur möglichen Eigenschaft von Meinungen, die wir über die Dinge in der Welt äußern und für die wir einen Geltungsanspruch erheben, den wir bei Bedarf überzeugend verteidigen bzw. einlösen müssen, um Zustimmung zu finden als Basis kooperativer Handlungschancen (Rorty 1991, $21 \mathrm{ff}.) .^{54}$

Damit ist die Grenze hinreichend markiert, die eine philosophische Rettung der Rhetorik in der Moderne von Aristoteles' gleichsinnigem Versuch unterscheidet, der wegen seiner ontologischen Denkvoraussetzungen über eine Wertschätzung von Rhetorik als Verlegenheitslösung kaum hinausdenken konnte. Doch ohne diese Grenze wieder aufweichen zu wollen, die zwischen der zur Zustimmung nötigenden Überzeugungskraft als Wahrheits- bzw. Geltungsindiz und ihrer Rolle als Wahrheits- bzw.

54 Vgl. Habermas 1984, 151 ff. zur „Verwechselung von Objektivität und Wahrheit“: „Wahrheit gehört kategorial der Welt der Gedanken an [...] und nicht der Wahrnehmung“; zur Unterscheidung zwischen „Wahrheit“ und „Richtigkeit“ s. 1999, 271 ff. und unten Kap. 10.2.2. 
Geltungskonstitution liegt, bleibt doch bemerkenswert, wie nah sich Aristoteles argumentativ dieser Grenze angenähert hat. Denn um einen zustimmungsabhängigen Wahrheits- bzw. Geltungsbegriff zu beschreiben, kann man sich fast komplett einer Definition bedienen, mit der Aristoteles den Überzeugungsbegriff bestimmt hat: „überzeugungskräftig [pithanon] ist etwas immer für jemanden“ (Rhet. 1356a). Ersetzt man „überzeugungskräftig“ durch „wahr“, dann wird „wahr“ zu einem Geltungsanspruch und damit an ein Referenzsubjekt als Bedingung seiner Geltungskonstitution gebunden: „Wahr“ ist etwas immer für jemanden, der einen entsprechenden Geltungsanspruch aufgrund einer ihn überzeugenden Einlösung bzw. unterstellten Einlösbarkeit zustimmend anerkennt. „Wahr“ wird also, was „überzeugungskräftig“ nach Aristoteles ist, nämlich ein „Relationsbegriff“ (Rapp 2002/1, 169), der seine ontologischen Fesseln gesprengt hat und alle Geltungsansprüche in einen Relations- bzw. Referenzsog zieht, vor dem Aristoteles noch sein TeleologieArgument schützen konnte, was aber nach der zur „kopernikanischen Wende“ nobilitierten Revolution, die sich mit den Namen Kant verbindet, kaum noch gelingen kann. Wir werden unten (Kap. 8), wenn wir über die kognitiven Voraussetzungen der Rhetorik reden, einen Brückenschlag von Kant (trotz seiner Rhetorik-Kritik!) über Gustav Gerber zu Nietzsches großen Satz „Die Sprache ist Rhetorik“ versuchen, der das Ergebnis eines kühnen Versuches war, Aristoteles' Rhetorik mit modernen Augen zu lesen. Darin drückt sich eine besondere Hochschätzung des Aristotelischen Textes aus, über den Nietzsche notiert, dass er im Vergleich zu anderen einschlägigen antiken Texten den Eindruck erwecke, „als habe es bis jetzt keine Rhetorik gegeben“ (KGA II 4, 521ff.). Ich kann diese Einschätzung Nietzsches bedenkenlos teilen, wenn ich auch anmerken muss, dass Nietzsche für die Geschichte der philosophischen Entdeckung dieser anderen Rhetorik weithin folgenlos geblieben ist, weshalb diese Entdeckung später auch noch einmal erfolgen musste, u. a. durch Perelman und Blumenberg. Aber auch deren Rezeption war - anders als im Fall Gadamer - so zögerlich, dass Habermas zunächst nicht einmal bemerkte, dass sich sein universalistisches Konzept einer Wahrheits- bzw. Geltungstheorie weithin mit Perelmans rhetorisch inspirierter Idee vom ,universalen Publikum“ (auditoire universel) deckte (s.u. Kap. 6). Nicht auszudenken, wie die rhetoriktheoretische Diskussion in Deutschland intensiviert und belebt worden wäre, hätte Habermas frühzeitig die Adresse dieser anderen, nämlich philosophisch ambitionierten Rhetorik erfahren, die man in Perelmans Brüssel - durch Zufall! - gefunden hatte und die Habermas selbst mit seiner äußerst rhetorikaffinen Terminologie unbewusst längst vielfach umschrieben hatte.

Wer wie ich die Attraktivität des Aristotelischen Konvergenztheorems, das die zur Zustimmung nötigende Überzeugungskraft einer Aussage als deren Wahrheitsindikation lesen will, unbedingt retten möchte, ohne zugleich die revitalisierungsunfähigen Denkvoraussetzungen dieses Theorems (etwa die Teleologie-Annahme) mitretten zu müssen, dem werden die beiden genannten Denker Perelman und 
Habermas hilfreiche Dienste anbieten können, wie an deren zustimmungsabhängiger Geltungstheorie noch zu zeigen sein wird (Kap. 6.4). Denn die bloße Faktizität einer Zustimmung kann schwerlich als Grund ausreichen, um darin das Sprachrohr der Vernunft vernehmen zu wollen. Und auch Aristoteles' zusätzliche charakteriologische Annahme (Aufrichtigkeit des Redenden, Rhet. 1356a) reicht als Zusatzbedingung nicht aus, um das Konvergenztheorem zu retten. Ebenso hat Quintilian zwar recht, dass sich der rhetorische Erfolg nicht simulieren lässt (IO XII 3.12), wohl aber lassen sich dessen normative Ermöglichungsbedingungen durchaus erfolgreich simulieren, ${ }^{55}$ weshalb eine redekritische (nicht redetheoretische) Unterscheidung zwischen „überreden“ und „überzeugen“ bzw. zwischen erfolgsund verständigungsorientiertem Redeinteresse unbedingt nötig ist (s. u. Kap. 11.3).

Festzuhalten bleibt also an Aristoteles' Aussagen über die Nützlichkeit der Rhetorik besonders dies: Rhetorik ist nützlich, weil sie das Wahre und Gerechte, kurz: das Vernünftige bzw. die Vernunft, statt sie im Interesse größerer Überzeugungskraft bloß zusätzlich noch beredt machen zu sollen, selbst zum Reden nötigt, um so deren inhärente Überzeugungskraft methodisch zustimmungswirksam zu entbinden. Rhetorik begibt sich damit auf einen Weg, der - um es mit der eben benutzten funktionalen Kurzformel zu sagen - zu ihrem Selbstverständnis als einer zustimmungsabhängigen Geltungstheorie führen wird und führen muss. ${ }^{56}$ Dieser Weg wird die Rhetorik zwar nicht völlig aus ihrer mängelkompensierenden Grundfunktion befreien, die sie als Konzessions- wie als Verlegenheitsrhetorik bei Platon bzw. Aristoteles an das bindet, was die defizitäre Struktur von Praxis psychologisch und/oder ontologisch ausmacht oder was man mit einem oben (Kap. 2) bereits genannten Ciceronianischen Begriff zusammenfassend auch die „Necessität“ (notwendiges Übel) des menschlichen Lebens nennen könnte. Doch diese „Necessität“ als normaler Raum der Rhetorik ist paradoxerweise zugleich ein Raum, in dem Menschen ihr Leben als „Freie und Gleiche“ leben können, weil gerade seine Mängelstruktur sie zu einem selbstbestimmten Leben nötigt, das sozial nur als ein verständigungsbezogenes Leben gelingen kann. Dass diese dialektische Form von Selbstbestimmung aber selbst wieder als „Necessität“ erfahren oder erlitten werden kann, ist - so viel Gegenwartsbezug sei erlaubt in Zeiten wie den jetzigen exemplarisch $\mathrm{zu}$ beobachten, wenn die Permanenz

55 Zum philosophischen Charakter der Aristotelischen Rhetorik vgl. ausführlich Rapp 2002/1, 378 ff.; im Kapitel Rhetorik bei Welsch, Wolfgang. Der Philosoph. München: Wilhelm Fink, 2012, $343 \mathrm{ff}$. merkt man leider wenig davon.

56 Der Vorteil der Habermas'schen Konzeption einer zustimmungsabhängigen Geltungstheorie liegt darin, dass die für einen Überzeugungserfolg notwendigen Idealisierungen anders als bei Aristoteles nicht an Personen festgemacht werden, sondern an Situationsbedingungen (,ideale Sprechsituation“), die zumindest schwerer als charakterliche Einstellungen bloß simulierbar sind. 
globalisierter Krisen einen Problemdruck erzeugt, der die von Aristoteles empfohlene deliberative Verständigungsarbeit politisch wie gesellschaftlich auf eine harte Probe stellt. Deliberation ist eben doch nicht nur eine rhetorische Kompensation menschlicher Mängel, sondern zugleich auch eine ihrer anstrengendsten Erscheinungsformen, weshalb sie Blumenberg u.a. sogar ein „Armutszeugnis“ zu nennen wagt. Dazu mehr im nächsten Kapitel! 